\title{
Desenvolvimento de um guia paciente-específico para fixação de coluna cervical alta*
}

\section{Development of a Patient-specific Guide for High Cervical Spine Fixation}

\author{
Felipe de Negreiros Nanni ${ }^{1}$ Emiliano Neves Vialle ${ }^{1}$ José Aguiomar Foggiattob ${ }^{2}$ \\ Kayo Winiccius Samuel Neves e Silva ${ }^{2}$ Heraldo de Oliveira Mello Neto ${ }^{3}$
}

\footnotetext{
${ }^{1}$ Hospital Universitário Cajuru, Pontifícia Universidade Católica do Paraná, Curitiba, PR, Brasil

${ }^{2}$ Núcleo de Prototipagem Ferramental, Departamento de Mecânica,

Universidade Tecnológica Federal do Paraná, Curitiba, PR, Brasil

${ }^{3}$ Serviço de Radiologia, X-Leme, Curitiba, PR, Brasil
}

Address for correspondence Felipe de Negreiros Nanni, Hospital Universitário Cajuru, Pontifícia Universidade Católica do Paraná, Curitiba, PR 80215-901, Brasil (e-mail: felipe_nanni@hotmail.com).

Rev Bras Ortop 2019;54:20-25.

\author{
Resumo \\ Palavras-chave \\ - vértebras cervicais/ \\ patologia \\ - vértebras cervicais/ \\ cirurgia \\ - fraturas da coluna \\ vertebral \\ - fusão vertebral \\ - impressão \\ tridimensional
}

Objetivos A fixação de coluna cervical alta pode representar um desafio para os cirurgiões de coluna devido à anatomia complexa e aos riscos de lesão vascular e medular. Os recentes avanços com a tecnologia de impressão $3 \mathrm{D}$ abriram um novo leque de opções para os cirurgiões.

Métodos Desenvolveu-se um guia para a adaptação de parafusos de massa lateral em C1 com auxílio de impressão 3 D. Foram confeccionados oito modelos em tamanho real de coluna cervical alta e seus respectivos guias com base em tomografias computadorizadas. Os fios-guia foram introduzidos com o auxílio dos guias; os modelos foram analisados com auxílio de tomografia computadorizada.

Resultados Todos os fios-guia avaliados no estudo apresentaram um trajeto seguro nos modelos, respeitaram as superfícies articulares superiores e inferiores, o canal vertebral e a artéria vertebral.

Conclusão O estudo demonstrou que o guia tem boa eficácia, é uma ferramenta confiável para auxiliar a adaptação de fios-guia para parafusos em massas laterais de C1.
Abstract
Objective High cervical spine fixation represents a challenge for spine surgeons due to the complex anatomy and the risks of vascular and medullar injury. The recent advances in 3-D printing have unfolded a whole new range of options for these surgeons.

\footnotetext{
*Trabalho desenvolvido no Hospital Universitário Cajuru, Curitiba, PR, Brasil. Publicado originalmente por Elsevier Editora Ltda. (C) 2018 Sociedade Brasileira de Ortopedia e Traumatologia.

(D) Felipe de Negreiros Nanni's ORCID is https://orcid.org/00000001-6959-8862.
}

received

August 16, 2017

accepted

September 5, 2017

published online

January 8, 2018
DOI https://doi.org/

10.1016/j.rbo.2017.09.011. ISSN 0102-3616.
Copyright $\odot 2019$ by Sociedade Brasileira License terms de Ortopedia e Traumatologia. Published by Thieme Revnter Publicações Ltda, Rio de Janeiro, Brazil 


\section{Keywords}

- cervical vertebrae/ pathology

- cervical vertebrae/ surgery

- spinal fractures

- spinal fusion

- three-dimensional printing
Methods In the present study, a guide for the placement of the lateral mass screw in the $\mathrm{C} 1$ vertebra was developed using 3-D printing. Eight real-size models of the high cervical spine and their respective screw guides were built using computed tomography (CT) scan images. The guidewires were inserted with the help of the printed guides and then the models were analyzed with the help of CT scan images.

Results All of the guidewires in the present study obtained a safe placement in the models, avoiding the superior and inferior articular surfaces, the vertebral foramen, and the vertebral artery.

Conclusion The present study demonstrated the efficiency of the guide, a reliable tool for aiding the insertion of guidewires for screws in lateral masses of the $\mathrm{C}$.

\section{Introdução}

A fixação de coluna cervical alta sempre foi um desafio para os cirurgiões de coluna. A anatomia complexa, a importância dessa região para a mobilidade cervical ${ }^{1}$ e a área relativamente pequena para fixação geram diversas dificuldades técnicas. ${ }^{2}$ Inicialmente os métodos de fixação eram insuficientes, como as técnicas de amarria de Brooks-Jenkins ou Gallie, $^{3}$ e requeriam fixação externa prolongada, ou eram extensas, incluíam a região occipital e limitavam significativamente a mobilidade cervical alta.

$\mathrm{O}$ advento da fixação com parafusos nas massas laterais, inicialmente com Goel e Laheri ${ }^{4}$ e depois com Harms e Melcher, ${ }^{5}$ mudou o espectro da cirurgia para coluna cervical alta. Entretanto, os desafios anatômicos persistem, principalmente pela localização da artéria vertebral lateral e superior ao ponto de entrada desses parafusos, ${ }^{6}$ pela artéria carótida anteriormente, pela medula espinhal medialmente ${ }^{7}$ e pelo extenso plexo venoso conjunto à raiz de $\mathrm{C} 2$ sobre o ponto de entrada do parafuso em $\mathrm{C} 1 .^{2}$ Ainda, esse procedimento requer o uso de radioscopia contínua, nociva à saúde tanto do paciente quanto da equipe médica. ${ }^{8}$

O uso de navegação computadorizada surgiu como uma opção para aumentar a acurácia desse método. ${ }^{9}$ Entretanto, apresenta custo elevado e não está disponível na maioria dos centros hospitalares.

Neste estudo os autores visam a oferecer uma opção de baixo custo e fácil acesso para reduzir os riscos associados à fixação cervical alta, através de um guia específico para a anatomia do paciente, confeccionado com o auxílio de impressão $3 \mathrm{D}$.

O objetivo foi avaliar a acurácia da fixação das massas laterais de $\mathrm{C} 1$ com o uso de um guia paciente-específico feito com impressora $3 \mathrm{D}$ através de um modelo cervical construído com impressão $3 \mathrm{D}$ e tomografias de controle.

\section{Material e Métodos}

\section{População}

A população do estudo compreende imagens tomográficas não identificadas de oito pacientes adultos com mais de 18 anos. Nenhum dos oito pacientes tinha alterações morfológicas extremas ou deformidades significativas na coluna cervical alta.

\section{Técnica Radiográfica}

Foram selecionadas tomografias computadorizadas de alta resolução com cortes finos $(01 \mathrm{~mm})$. Os filmes foram analisados pelos autores, constataram-se a ausência de deformidades importantes e a presença de pontos anatômicos importantes preservados.

\section{Confecção dos Modelos de Coluna Cervical Alta}

A partir das imagens bidimensionais sequenciais da tomografia, com o auxílio do programa InVesalius (CTI, Campinas, $\mathrm{SP}$ ), os exames são convertidos para um modelo tridimensional, que então é exportado para o programa CAD $3 \mathrm{D}$ Meshmixer (Autodesk Inc, San Rafael, CA) e então manipulado de forma a isolar apenas C1, C2 e parcialmente C3.

Os modelos de vértebras cervicais foram então impressos em ABS na impressora 3 D Vantage I (Stratasys, Inc, Eden Prairie, MN) em escala 1:1 (- Fig. 1).

\section{Confecção dos Guias Para Fixação Pedicular}

Com os modelos de coluna cervical finalizados, foi criado um guia para parafusos pediculares, idealizado de forma que o guia se adaptasse de maneira estável sobre as vértebras, com limitação para translação lateral ou supero inferior e com vistas a evitar estruturas anatomicamente importantes.

Com auxílio do programa CAD 3 D Meshmixer (Autodesk Inc, San Rafael, CA), o modelo virtual do guia foi então montado sobre cada reconstrução virtual de cervical alta individualmente. Através de operação boleana identificou-se a interseção entre os modelos e ajustes finos foram feitos individualmente para que os guias se encaixassem adequadamente nas superfícies posteriores de todas as colunas cervicais ava liadas.

Os trajetos de cada guia foram definidos individualmente com auxílio do cirurgião, simularam por computador qual o trajeto desejado para cada parafuso.

Os modelos dos guias foram então impressos em resina acrílica Fullcure 720 pela impressora Objet EDEN250 (Stratasys, Eden Prairie, MN) (- Fig. 2).

\section{Adaptação dos Fios-guia}

Os guias impressos foram adaptados sobre os respectivos modelos impressos de coluna cervical. Verificou-se o encaixe perfeito entre os modelos de cervical e os guias e demonstrou-se a estabilidade do guia ao manuseio. 


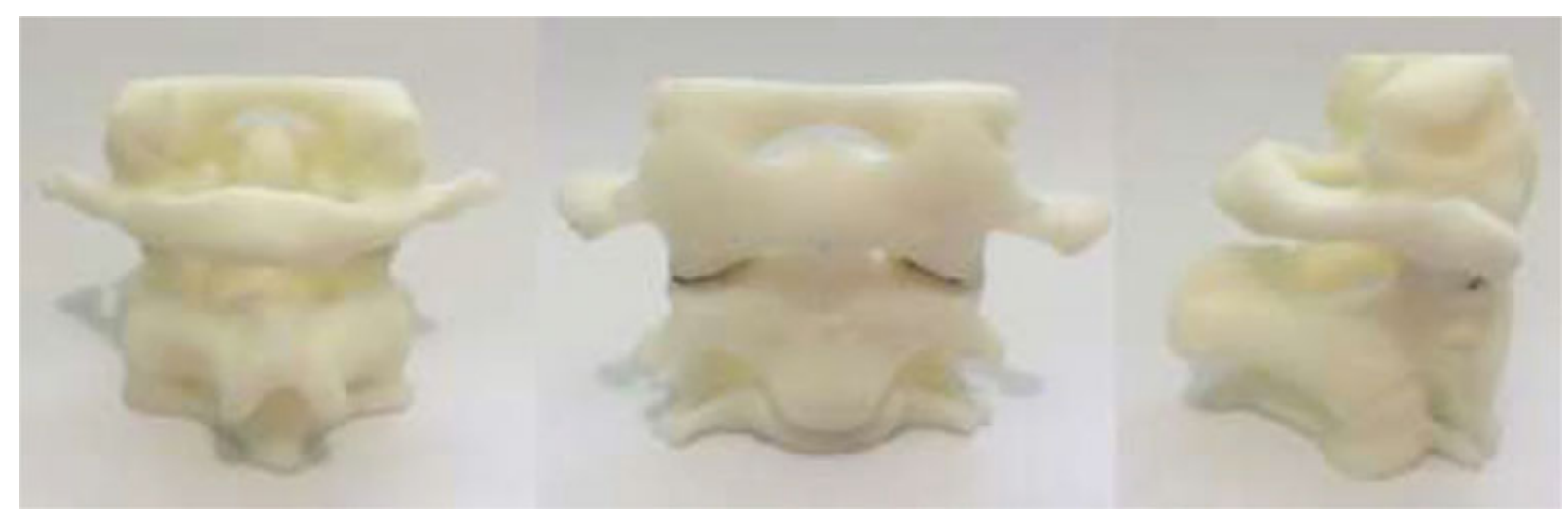

Fig. 1 Modelo de coluna cervical alta impresso em resina plástica.

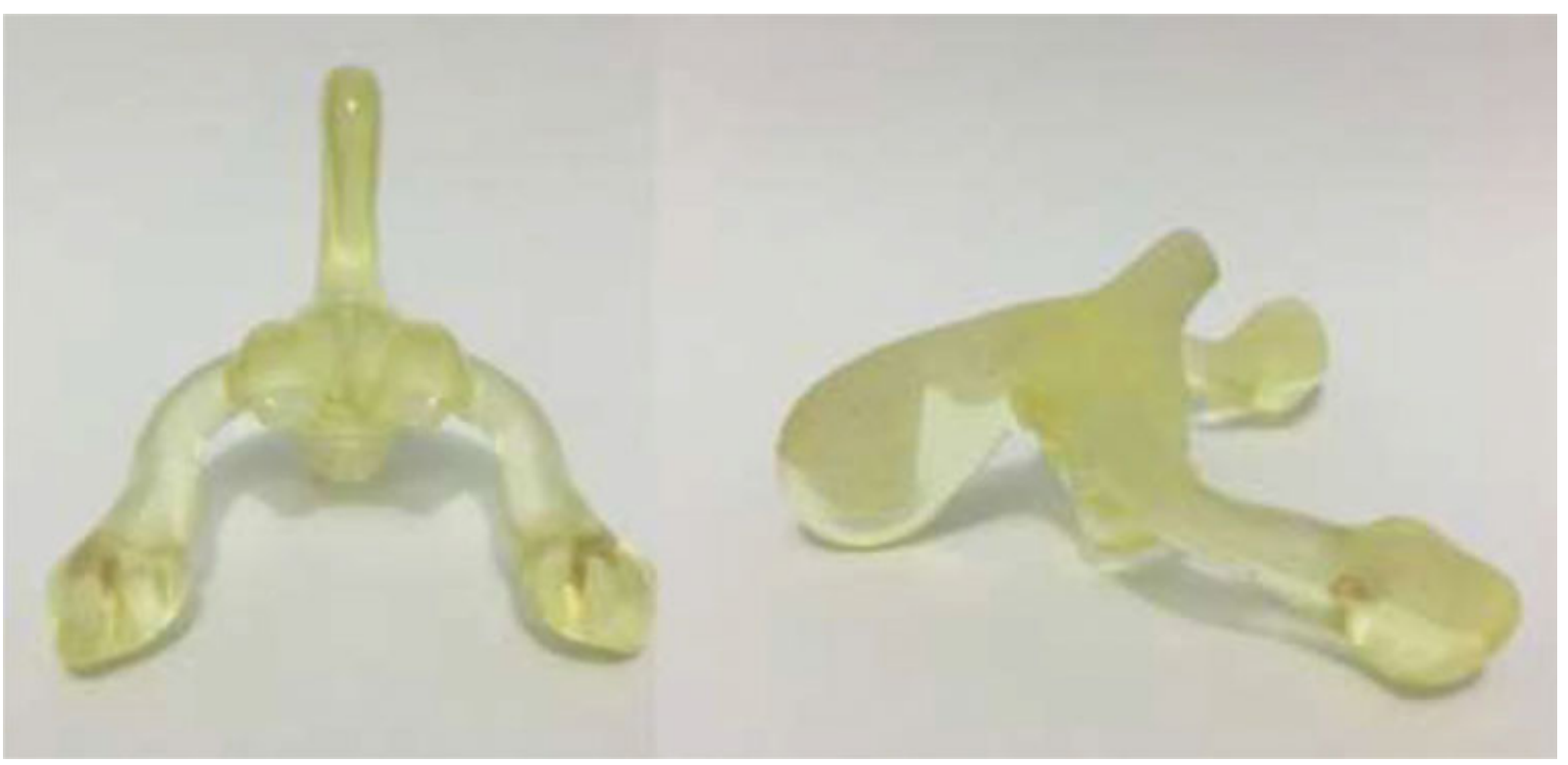

Fig. 2 Guia para inserção de fios guia nas massas laterais de $C 1$ impresso em resina acrílica.

Com os modelos firmemente seguros com o auxílio de uma morsa e os guias adaptados e firmemente posicionados pelo cirurgião, foram feitas perfurações com broca número $1.5 \mathrm{~mm}$ em ambos os pedículos das oito vertebras C1. Após a perfuração a broca era solta do perfurador e permanecia afixada ao modelo.

Após feitas as 16 perfurações, os guias foram removidos e as peças foram então enviadas a um serviço de diagnóstico por imagem para feitura de tomografia computadorizada com cortes finos $(01 \mathrm{~mm})$ (-Figs. 3 e 4).

\section{Análise dos Resultados}

Após obtidas as imagens das peças com os fios guias adapta dos, foi feita a mensuração do posicionamento dos fios guia com o auxílio do programa RadiAnt DICOM Viewer (Medixant, Poznan) com base em quatro parâmetros: distância do fio guia ao forame da artéria vertebral; distância do fio guia ao canal medular; distância do fio guia à superfície articular superior de $\mathrm{C} 1$ e distância do fio guia à superfície articular inferior de C1 (-Fig. 5).

\section{Resultados}

Na avaliação das imagens tomográficas obtidas, constatou-se que nenhum dos 16 fios guia nos oito modelos do estudo invadiu a área do canal medular ou as superfícies articulares, tampouco se encontrava na região do trajeto da artéria vertebral.

A distância do forame da artéria vertebral variou de 2,8 a 6,2 $\mathrm{mm}$, com valor médio de $4,08 \mathrm{~mm}$, mediana de $4 \mathrm{~mm}$ e desvio-padrão de $0,937 \mathrm{~mm}$.

A distância do canal vertebral variou de 3,7 a $8 \mathrm{~mm}$, com valor médio de $5,83 \mathrm{~mm}$, mediana de $6 \mathrm{~mm}$ e desvio-padrão de $1,266 \mathrm{~mm}$.

A distância do fio guia à superfície articular superior de $\mathrm{C} 1$ variou de 5,9 a $10,7 \mathrm{~mm}$, com valor médio de $7,52 \mathrm{~mm}$, mediana de 7,1 mm e desvio-padrão de $1,212 \mathrm{~mm}$.

A distância do fio guia à superfície articular inferior de C1 variou de 2 a 5,9 $\mathrm{mm}$, com valor médio de $3,62 \mathrm{~mm}$, mediana de $3,5 \mathrm{~mm}$ e desvio-padrão de $0,988 \mathrm{~mm}$.

Os valores de cada parâmetro estão listados na - Tabela 1. 


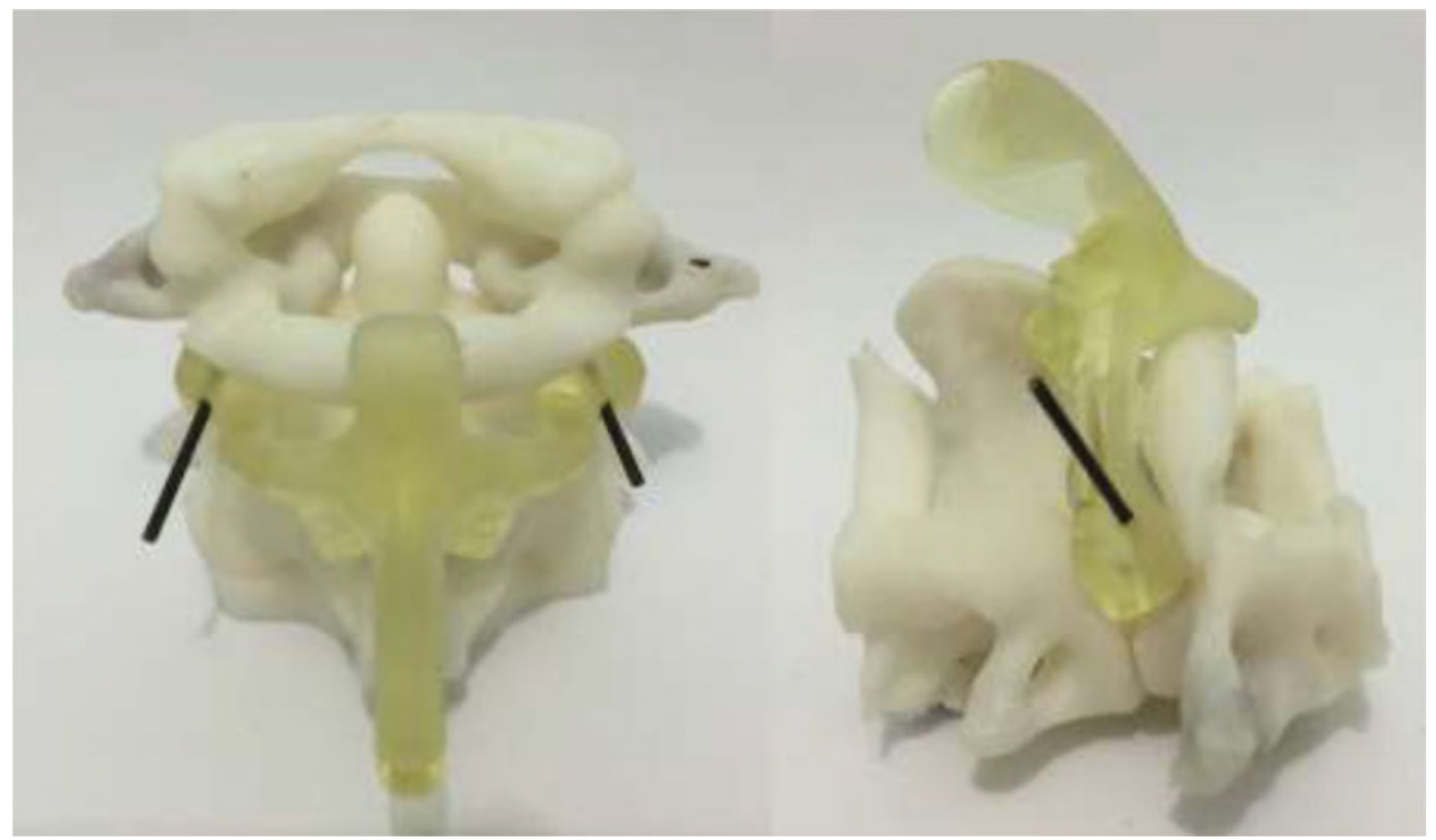

Fig. 3 Guia adaptado sobre a vértebra com a perfuração com fio-guia já feita.

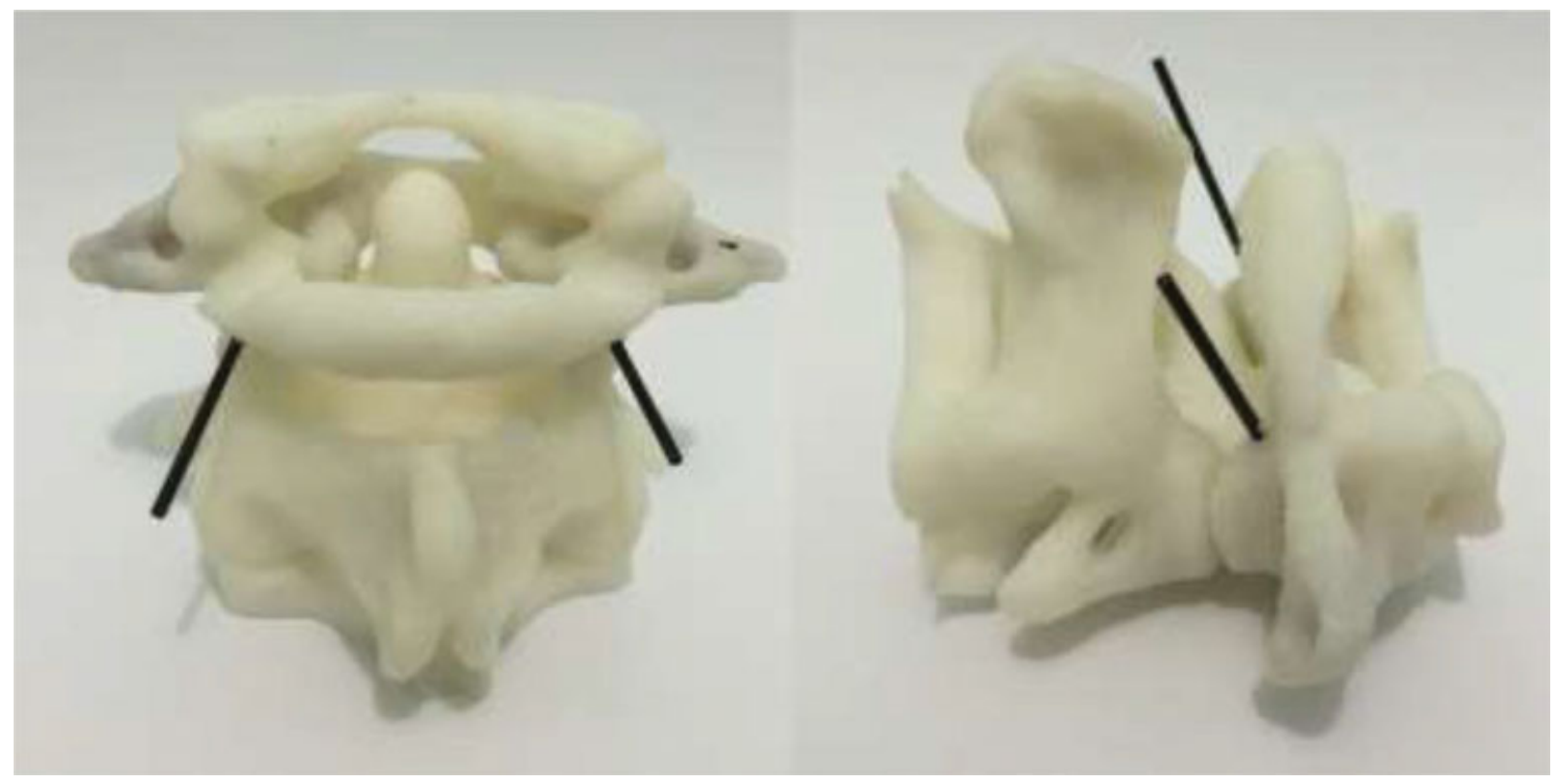

Fig. 4 Modelo após a retirada do guia impresso.

\section{Discussão}

A impressão $3 \mathrm{D}$ tem se mostrado um recurso promissor no auxílio do planejamento e da execução de cirurgias complexas de coluna, permite recriar com grande acurácia modelos anatômicos complexos a partir de exames de imagem. ${ }^{10-12}$

Essa técnica já é usada com sucesso para estudos de anatomia, planejamentos cirúrgicos e até mesmo na criação de implantes biocompatíveis. ${ }^{13-15}$
Trabalhos recentes têm investigado a eficácia do uso da impressão 3 D na confecção de guias cirúrgicos específicos para o paciente que auxiliam na adaptação de parafusos pediculares em vértebras da coluna lombar e torácica. Já existem evidências de bons resultados até mesmo com o uso intraoperatório desses guias in vivo.

Em um experimento feito por Fu et al., ${ }^{16}$ guias de polimetilmetacrilato para parafusos transpediculares foram moldados manualmente sobre modelos de vértebras cervicais impressos com impressora $3 \mathrm{D}$ e testados em vértebras 


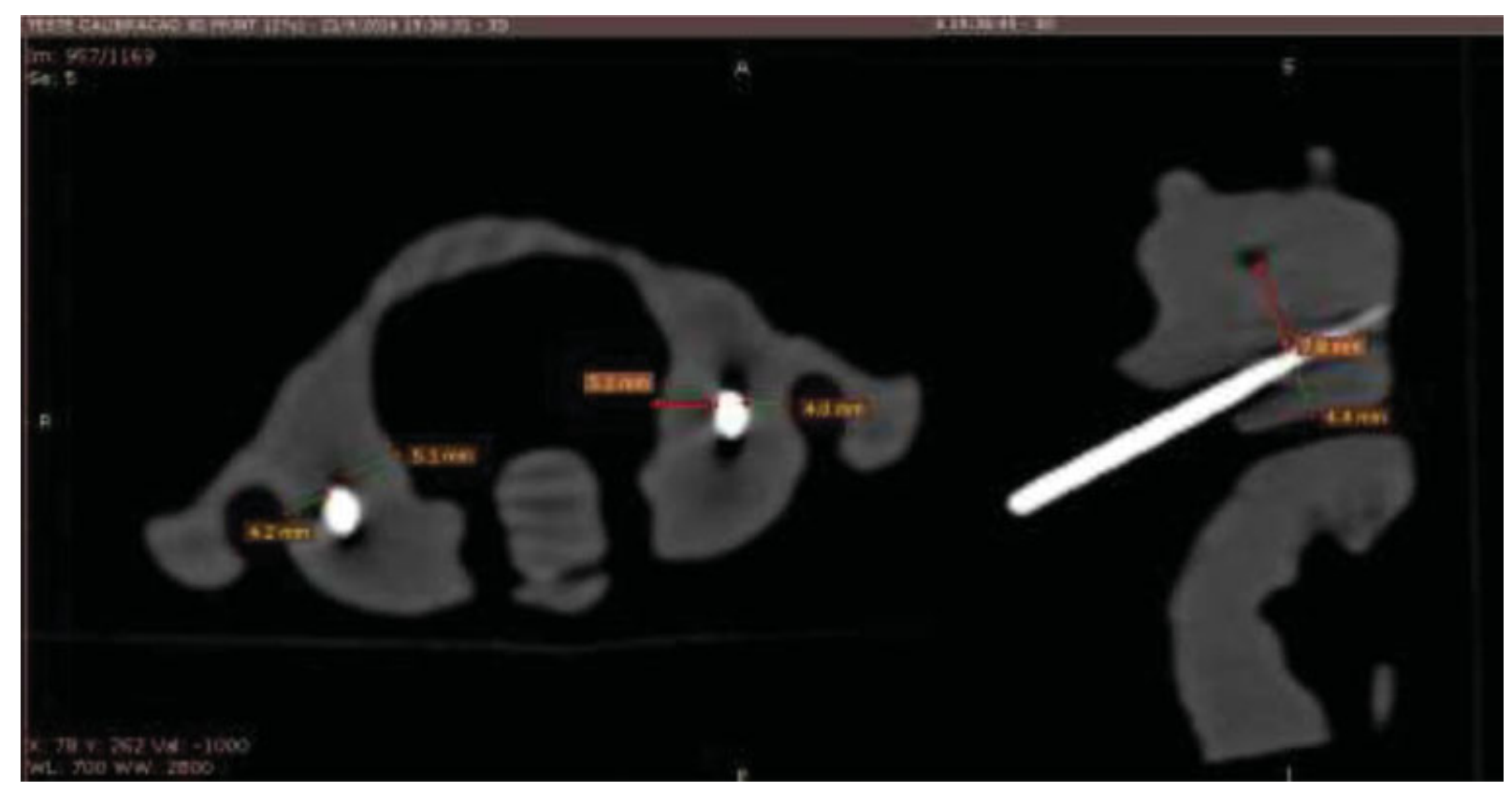

Fig. 5 Aferição dos parâmetros anatômicos das tomografias computadorizadas.

Tabela 1 Parâmetros dos modelos experimentais

\begin{tabular}{|l|l|l|l|l|l|l|l|l|}
\hline & Modelo 01 & Modelo 02 & Modelo 03 & Modelo 04 & Modelo 05 & Modelo 06 & Modelo 07 & Modelo 08 \\
\hline DCVD & 6,1 & 6,6 & 7,4 & 3,7 & 8,0 & 6,1 & 5,4 & 5,1 \\
\hline DCVE & 5,1 & 6,3 & 4,5 & 3,8 & 7,7 & 6,0 & 6,4 & 5,2 \\
\hline DAVD & 4,4 & 3,4 & 3 & 3,5 & 2,8 & 3,6 & 4,0 & 4,2 \\
\hline DAVE & 5,1 & 3,3 & 6,2 & 5,7 & 4,2 & 4,4 & 3,5 & 4,0 \\
\hline DSASD & 6,7 & 5,9 & 8,3 & 6,6 & 10,7 & 4,4 & 7,0 & 7,7 \\
\hline DSASE & 7,2 & 6,8 & 8,6 & 6,7 & 9,2 & 4,0 & 6,6 & 7,6 \\
\hline DSAID & 4,0 & 3,2 & 4,2 & 2,0 & 2,8 & 7,8 & 2,6 & 5,1 \\
\hline DSAIE & 3,0 & 3,0 & 3,9 & 3,0 & 3,8 & 8,0 & 3,1 & 5,9 \\
\hline
\end{tabular}

Abreviações: DAVD, distância da artéria vertebral à direita; DAVE, distância da artéria vertebral à esquerda; DCVD, distância do canal vertebral à direita; DCVE, distância do canal vertebral à esquerda; DSAIE, distância da superfície articular inferior à esquerda; DSASD, distância da superfície articular superior à direita; DSASE, distância da superfície articular superior à esquerda; DSAID, distância da superfície articular inferior à direita.

de cadáveres, obtiveram-se bons resultados e demonstrou-se fácil aplicabilidade.

Alguns estudos também foram feitos para desenvolver guias de parafusos pediculares para vértebras cervicais impressos diretamente na impressora $3 \mathrm{D}$, obtiveram excelentes resultados. Em um experimento executado por Sugawara et al., ${ }^{17} 100 \%$ dos 80 parafusos adaptados em 20 pacientes obtiveram uma localização precisa de acordo com a trajetória planejada no pré-operatório, com um desvio médio de $0,29 \pm 0,31 \mathrm{~mm}(0,0 \mathrm{~mm}-1,6 \mathrm{~mm})$.

Ainda são poucos os estudos relativos ao uso da impressão $3 \mathrm{D}$ na cirurgia da coluna vertebral e até o presente momento não se acha na literatura um estudo consistente a respeito do uso desses guias pediculares para $\mathrm{C} 1$.

A região atlantoaxial consiste em um desafio cirúrgico, com propriedades anatômicas e biomecânicas únicas. Diversas técnicas foram desenvolvidas, porém na última década ganhou destaque a fixação com parafusos em massa lateral de C1. ${ }^{18}$

Existem dificuldades técnicas para identificar e acessar o ponto ideal de entrada do parafuso de massa lateral em C1. Por essa razão um planejamento pré-operatório cuidadoso é necessário para evitar mau posicionamento dos parafusos e demasiada exposição que pode levar a um sangramento expressivo e prejudicar o sucesso da cirurgia. ${ }^{18,19}$

Entre essas dificuldades durante 0 acesso à face posterior da massa lateral de C1 estão o sangramento abundante encontrado ao expor-se a face inferior do arco posterior e da parte posterior da massa lateral de $\mathrm{C}^{20}$ e o risco de lesão da artéria vertebral ao mobilizá-la no sulco do arco de $\mathrm{C} 1{ }^{6}$

Devem ser feitas a exposição do nervo C2 e a dissecção cuidadosa dos tecidos adjacentes ao gânglio dorsal da raiz de $\mathrm{C} 2 .^{6}$ 
Os parafusos de massa lateral em C1 são inseridos diretamente na massa lateral, inferiormente à base do arco posterior. As dimensões da massa lateral de C1 são capazes de acomodar facilmente parafusos de $3,5 \mathrm{~mm}$ na maioria dos pacientes. $^{19}$

De acordo com a técnica descrita por Harms e Melcher, ${ }^{5}$ os parafusos devem ser inseridos na direção posteroanterior, com 5o-10॰ de convergência no plano axial. No plano sagital devem permanecer paralelos ao aspecto caudal do arco posterior de $\mathrm{C} 1$, apontar para o centro do tubérculo anterior de $\mathrm{C} 1{ }^{6}$

Durante os estudos para a preparação dos guias através do computador, esses aspectos anatômicos foram cuidadosamente observados de modo a definir um trajeto ideal individualizado para cada modelo do estudo.

$\mathrm{O}$ ineditismo do estudo levou a algumas dificuldades quanto ao formato ideal do guia, diferentes modelos de guia foram confeccionados e testes foram feitos para verificar qual modelo de guia apresentaria maior estabilidade quando adaptado aos modelos de vértebra respectivos.

Ao se avaliarem os valores numéricos obtidos para cada vértebra, foram levadas em consideração as diferenças anatômicas de morfologia e de tamanho presentes entre os oito modelos do experimento.

Em nenhum dos oito modelos os fios-guia invadiram a região articular, o canal medular ou o trajeto da artéria vertebral. Todos os 16 fios guia foram passados uma única vez, sem falsos trajetos.

Todos os fios-guia adaptados mantiveram o trajeto através do centro das massas laterais de C1 de maneira satisfatória, apresentaram massa óssea adjacente suficiente para uma possível adaptação de um parafuso canulado com segurança.

Durante as perfurações o guia se mostrou resistente e não houve caso de quebra do guia impresso ou deformação do material com o calor gerado pela broca. Uma pequena quantidade de debris provenientes dos guias foi observada após as perfurações. Tendo em vista que a literatura atual não dispõe de dados conclusivos quanto ao efeito desses sobre organismos vivos em longo prazo, estudos adicionais devem ser conduzidos.

Tendo em vista os bons resultados obtidos com os guias, novos estudos serão feitos com o âmbito de aperfeiçoar os guias e verificar sua possível aplicabilidade in vivo.

\section{Conclusão}

O experimento demonstrou que os guias paciente-específicos confeccionados com auxílio de impressora $3 \mathrm{D}$ permitiram o posicionamento de fios guia na massa lateral de C1 com $100 \%$ de precisão. Esses guias têm fácil aplicação, podem trazer benefícios para a adaptação de parafusos em massa lateral de $\mathrm{C} 1$ e fornecer um trajeto seguro para os parafusos.

Conflitos de Interesse

Os autores declaram não haver conflitos de interesse.

\section{Referências}

1 Swartz EE, Floyd RT, Cendoma M. Cervical spine functional anatomy and the biomechanics of injury due to compressive loading. J Athl Train 2005;40(3):155-61

2 Abumi K, Shono Y, Ito M, Taneichi H, Kotani Y, Kaneda K. Complications of pedicle screw fixation in reconstructive surgery of the cervical spine. Spine 2000;25(8):962-9

3 Mummaneni PV, Haid RW. Atlantoaxial fixation: overview of all techniques. Neurol India 2005;53(4):408-15

4 Goel A, Laheri V. Plate and screw fixation for atlanto-axial subluxation. Acta Neurochir (Wien) 1994;129(1-2):47-53

5 Goel A, Laheri V. Re: Harms J, Melcher P. Posterior C1-C2 fusion with polyaxial screw and rod fixation. (Spine 2001;26: 2467-71). Spine 2002;27(14):1589-90

6 Schulz R, Macchiavello N, Fernández E, Carredano X, Garrido O, Diaz J, et al. Harms C1-C2 instrumentation technique: anatomosurgical guide. Spine 2011;36(12):945-50

7 Currier BL, Maus TP, Eck JC, Larson DR, Yaszemski MJ. Relationship of the internal carotid artery to the anterior aspect of the C1 vertebra: implications for $\mathrm{C} 1-\mathrm{C} 2$ transarticular and $\mathrm{C} 1$ lateral mass fixation. Spine 2008;33(6):635-9

8 Mettler FA Jr, Koenig TR, Wagner LK, Kelsey CA. Radiation injuries after fluoroscopic procedures. Semin Ultrasound CT MR 2002;23 (5):428-42

9 Amiot LP, Lang K, Putzier M, Zippel H, Labelle H. Comparative results between conventional and computer-assisted pedicle screw installation in the thoracic, lumbar, and sacral spine. Spine 2000;25(5):606-14

10 Wu AM, Shao ZX, Wang JS, Yang XD, Weng WQ Wang XY, et al. The accuracy of a method for printing three-dimensional spinal models. PLoS One 2015;10(4):e0124291

11 Yang M, Li C, Li Y, Zhao Y, Wei X, Zhang G, et al. Application of 3D rapid prototyping technology in posterior corrective surgery for Lenke 1 adolescent idiopathic scoliosis patients. Medicine (Baltimore) 2015;94(8):e582

12 Mizutani J, Matsubara T, Fukuoka M, Tanaka N, Iguchi H, Furuya A, et al. Application of full-scale three-dimensional models in patients with rheumatoid cervical spine. Eur Spine J 2008;17(5):644-9

13 Whatley BR, Kuo J, Shuai C, Damon BJ, Wen X. Fabrication of a biomimetic elastic intervertebral disk scaffold using additive manufacturing. Biofabrication 2011;3(1):015004

14 Xu N, Wei F, Liu X, Jiang L, Cai H, Li Z, et al. Reconstruction of the upper cervical spine using a personalized 3D-printed vertebral body in an adolescent with Ewing sarcoma. Spine 2016;41(1):E50-4

15 Wu AM, Wang S, Weng WQ, Shao ZX, Yang XD, Wang JS, et al. The radiological feature of anterior occiput-to-axis screw fixation as it guides the screw trajectory on 3D printed models: a feasibility study on 3D images and 3D printed models. Medicine (Baltimore) 2014;93(28):e242

16 Fu M, Lin L, Kong X, Zhao W, Tang L, Li J, et al. Construction and accuracy assessment of patient-specific biocompatible drill template for cervical anterior transpedicular screw (ATPS) insertion: an in vitro study. PLoS One 2013;8(1):e53580

17 Sugawara T, Higashiyama N, Kaneyama S, Takabatake M, Watanabe $\mathrm{N}$, Uchida F, et al. Multistep pedicle screw insertion procedure with patient-specific lamina fit-and-lock templates for the thoracic spine: clinical article. J Neurosurg Spine 2013;19(2):185-90

18 Wang MY, Samudrala S. Cadaveric morphometric analysis for atlantal lateral mass screw placement. Neurosurgery 2004;54(6): 1436-9, discussion 1439-40

19 Ma XY, Yin QS, Wu ZH, Xia H, Liu JF, Zhong SZ. Anatomic considerations for the pedicle screw placement in the first cervical vertebra. Spine 2005;30(13):1519-23

20 Christensen DM, Eastlack RK, Lynch JJ, Yaszemski MJ, Currier BL. $\mathrm{C} 1$ anatomy and dimensions relative to lateral mass screw placement. Spine 2007;32(8):844-8 